\title{
A Sweet Introduction to the Mathematical Analysis of Time-Resolved Spectra and Complex Kinetic Mechanisms: The Chameleon Reaction Revisited
}

\author{
Ricardo J. Fernández-Terán, ${ }^{a *}$ Estefanía Sucre-Rosales, ${ }^{b}$ Lorenzo Echevarria, ${ }^{b c}$ and Florencio E. Hernández ${ }^{b d}$ \\ ${ }^{a}$ Department of Chemistry, University of Sheffield. Sheffield S3 7HF, United Kingdom. \\ ${ }^{b}$ Department of Chemistry, University of Central Florida, Orlando, Florida 32816, United States. \\ ${ }^{c}$ Departamento de Química, Universidad Simón Bolivar, Caracas 1080-A, AP 89000, Venezuela. \\ ${ }^{d}$ CREOL/The College of Optics and Photonics, University of Central Florida, Orlando, Florida 32816, United \\ States. \\ *Corresponding Author: Ricardo.Fernandez@sheffield.ac.uk and Ricardo.FernandezTeran@gmail.com
}

(Dated: 5th February 2022)

\begin{abstract}
We present a detailed yet easy-to-follow discussion of the mathematical treatment of time-resolved spectroscopic data in a model-based approach. This is accompanied and complemented by an example of a colourful and pedagogically rich chemical reaction: the permanganate oxidation of sugars in basic aqueous media (often known as the chameleon reaction). Our sweet approach allows both students and lecturers to tackle the problem of a model-based analysis of time-resolved data in a simple manner and one step at the time, while also exercising programming and data analysis skills - fundamental for present and future chemists of all levels.
\end{abstract}

\section{INTRODUCTION}

Understanding the rates and mechanisms of chemical reactions is a matter of central importance in chemistry, biochemistry and other fields. Chemists can monitor the twists and turns of the reactant on its way to the product, and the kinetic or rate constants of each step using time-resolved spectroscopy. Careful analysis of a series of spectra collected at different reaction times ultimately provides the spectroscopic signatures and rate constants of the proposed intermediates, confirming the mechanistic picture. Armed with this knowledge, for instance, the reaction conditions can be optimised to avoid side reactions and optimise product yield - as is commonly done in industry or in the lab.

The treatment of time-resolved spectra in the context of ultrafast spectroscopy has been described in vast detail by van Stokkum et al., who popularised the method of global and target analysis. ${ }^{1}$ Other authors have published alternative data analysis implementations, including lifetime density analysis, ${ }^{2}$ and multivariate curve resolution by alternating least squares (MCR-ALS) ${ }^{3-6}$ A recent review by Vauthey et al. in the context of ultrafast transient UV-Vis and fluorescence upconversion spectroscopy summarises these methods in detail. ${ }^{7}$

Apart from specific details intrinsic to the treatment of ultrafast transient spectra, these analysis methods are general and independent of the timescale of the studied processes, motivating us to introduce it to chemistry students at any stage of their education. In the typical undergraduate chemistry curriculum, most emphasis is typically given to first- and second-order kinetics. ${ }^{8}$ More complex cases are often neglected or treated at an approximate level (e.g. by using the steady-state approximation), and approximate kinetic equations are often derived for certain limiting cases. A more advanced treatment involves numerical integration of the complex sys- tems of ordinary differential equations (ODEs) that arise in chemical kinetics. ${ }^{9-11}$ These systems, however, are often stiff, ${ }^{12}$ which can lead to numerically inaccurate solutions. An exhaustive description of numerical methods to solve systems of ODEs can be found elsewhere. ${ }^{13,14}$

In our previous work, ${ }^{15,16}$ we established an analogy between viral spread during a pandemic and chemical kinetics, using it to introduce more complex reaction mechanisms and abstract concepts - such as the collision cross section. Other authors also developed analogies with chemical kinetics concepts, ${ }^{17-20}$ and illustrated their power in helping the students master the fundamentals. Some of us have also applied complex kinetic schemes to model, for example, electron transfer processes in surfaceanchored molecular catalysts and photosensitisers for water splitting, ${ }^{21,22}$ proton-coupled electron transfer processes in tungsten carbonyls, where a dimerisation takes place after the initial event; ${ }^{23}$ and compared different strategies for the analysis of time-resolved data of protein non-equilibrium dynamics. ${ }^{24}$

Herein, we present a combined theoretical and practical treatment of more complex reaction mechanisms, exemplified by the oxidation of a carbohydrate in presence of $\mathrm{KMnO}_{4}$ - often called the chemical chameleon reaction. This colourful reaction can be readily monitored by UV-Vis absorption spectroscopy, and performed using inexpensive and readily available chemicals (Figure 1).

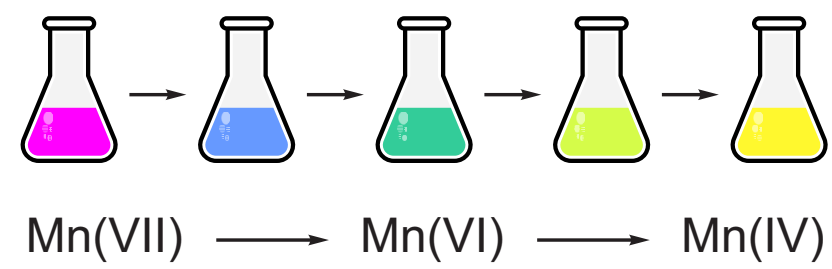

Figure 1. Sequence of colour changes and oxidation states of Mn observed during the chemical chameleon reaction. 
We have chosen the chemical chameleon reaction because of its simplicity, the availability of the required reagents, and the captivating colour changes that take place - a fact that we will use to our advantage when discussing the UV-Vis spectra. The chemical chameleon reaction is a widely performed demonstrative experiment in introductory chemistry labs, and can be used to teach fundamental concepts of redox chemistry. ${ }^{25-30}$ In this reaction, a carbohydrate is oxidised by the permanganate ion in an alkaline aqueous medium. The rapid colour changes are evidence of the different oxidation states of manganese - going from pink-violet $\mathrm{Mn}(\mathrm{VII})$, to green $\mathrm{Mn}(\mathrm{VI})$, and finally to yellow $\mathrm{Mn}(\mathrm{IV})$, as seen in Figure 1.

The chameleon reaction can be easily monitored with the naked eye, and a series of UV-Vis absorption spectra can be recorded for more quantitative spectrokinetic analysis. The time-dependent absorption spectra obtained from such experiments can be interpreted and fitted to a mechanistic model to further the students' understanding of key concepts in chemical kinetics and inorganic chemistry. The students will learn to carefully analyse and interpret time-dependent spectroscopic data, allowing them to extract rate constants and the spectroscopic signatures of the proposed intermediates in a complex reaction mechanism. The methods presented herein are general and can be adapted to specific mechanisms and to the study of other chemical reactions (by changing the kinetic/mechanistic model). Moreover, and as mentioned above, this approach can be adapted to all timescales - ranging from slow to ultrafast - and to other spectroscopic techniques - such as IR or NMR. Thanks to this, the students can even connect with the latest developments in ultrafast spectroscopy, obtaining tools to analyse the fastest chemical events by making analogies with slower reactions that can be monitored even with the naked eye.

\section{THEORETICAL BACKGROUND}

In this section we present a detailed mathematical treatment of chemical kinetics and spectrokinetic modelling, including advanced concepts of linear algebra and differential equations. This is provided herein in a tutorial manner to allow the interested reader to gain a deep understanding of the methods and the equations behind the analysis of time-resolved spectra.

\section{A. Initial Considerations}

We start from a simple chemical reaction, in which species $\mathrm{A}$ and $\mathrm{B}$ are converted into $\mathrm{X}$ and $\mathrm{Y}$, with their stoichiometric coefficients (denoted with the corresponding lowercase letters), as shown in eq. 1:

$$
a \mathrm{~A}+b \mathrm{~B} \longrightarrow x \mathrm{X}+y \mathrm{Y}
$$

The rate law of this reaction $(r)$ is given by:

$$
r=k[\mathrm{~A}]^{a}[\mathrm{~B}]^{b},
$$

where the brackets denote concentrations of the species, and the powers represent the stoichiometric coefficients of the reaction and are called order of the reaction (in terms of a given reactant). The total order of the reaction is thus the sum the reaction orders of all reactants.

The same rate law can be interpreted as the total rate of change of a reactant or product over time (eq. 3):

$$
r=-\frac{1}{a} \frac{d[\mathrm{~A}]}{d t}=-\frac{1}{b} \frac{d[\mathrm{~B}]}{d t}=\frac{1}{x} \frac{d[\mathrm{X}]}{d t}=\frac{1}{y} \frac{d[\mathrm{Y}]}{d t}
$$

Combining eq. 2 and eq. 3, we arrive to a differential equation of the form:

$$
\frac{d[\mathrm{~A}]}{d t}=a \times k[\mathrm{~A}]^{a}[\mathrm{~B}]^{b}
$$

which can be integrated to yield the analytical time dependence of the concentration of each participating species. For simple elementary reaction steps this is a trivial matter, and is often done in introductory chemical kinetics courses. ${ }^{8}$

A more complex case, for example, arises whenever there are several coupled reaction steps. For example, let us now consider an equilibrium followed by an irreversible reaction (e.g. a decomposition reaction, eq. 5):

$$
\mathrm{A} \underset{k_{-1}}{\stackrel{k_{1}}{\rightleftharpoons}} \mathrm{B} \stackrel{k_{2}}{\longrightarrow} \mathrm{X}
$$

In this case, the time dependence of the concentrations can no longer be derived based on a single differential equation (as in eq. 4). Instead, we must solve a system of three coupled ordinary differential equations (eq. 6) with three unknowns $\left([\mathrm{A}]_{t},[\mathrm{~B}]_{t}\right.$, and $[\mathrm{C}]_{t}$, where the subscript $t$ refers to the concentration at any time):

$$
\left\{\begin{array}{l}
\frac{d[\mathrm{~A}]_{t}}{d t}=-k_{1}[\mathrm{~A}]_{t}+k_{-1}[\mathrm{~B}]_{t} \\
\frac{d[\mathrm{~B}]_{t}}{d t}=+k_{1}[\mathrm{~A}]_{t}-k_{-1}[\mathrm{~B}]_{t}-k_{2}[\mathrm{~B}]_{t} \\
\frac{d[\mathrm{X}]_{t}}{d t}=+k_{2}[\mathrm{~B}]_{t}
\end{array}\right.
$$

We will focus on reactions in which all stoichiometric coefficients are equal to 1 , for which an analytical solution exists and is discussed next. More complex kinetic models have been discussed elsewhere. ${ }^{15,31,32}$

\section{B. Generalised Matrix Kinetics for First Order Reaction Systems}

The problem of solving systems of coupled firstorder reactions with matrix methods has been previously treated in detail in this Journal by Berberan-Santos and 
Martinho, ${ }^{31}$ and by others. ${ }^{11,33}$ We shall restate some of the concepts and methods in this paper so the reader can have a complete picture.

We start by designating a species concentration vector, $\boldsymbol{C}(t)=\left[\begin{array}{lll}\mathrm{A}(t) & \mathrm{B}(t) & \mathrm{X}(t)\end{array}\right]^{\mathrm{T}}$. This way, eq. 6 can be expressed in matrix form as:

$$
\frac{d}{d t}\left(\begin{array}{l}
{[\mathrm{A}]_{t}} \\
{[\mathrm{~B}]_{t}} \\
{[\mathrm{X}]_{t}}
\end{array}\right)=\left(\begin{array}{ccc}
-k_{1} & k_{-1} & 0 \\
k_{1} & -k_{-1}-k_{2} & 0 \\
0 & k_{2} & 0
\end{array}\right)\left(\begin{array}{l}
{[\mathrm{A}]_{t}} \\
{[\mathrm{~B}]_{t}} \\
{[\mathrm{X}]_{t}}
\end{array}\right)
$$

More generally, for any first-order system:

$$
\frac{d}{d t} \boldsymbol{C}(t)=\boldsymbol{K} \cdot \boldsymbol{C}(t)
$$

where $\boldsymbol{K}$ is an $N \times N$ matrix (for a system with $N$ species), often called the transition or rate matrix, and contains all the kinetic information about the model.

Since in eq. 8 we have written $\boldsymbol{C}(t)$ as a column vector, the off-diagonal elements of $\boldsymbol{K},\{\boldsymbol{K}\}_{i j}(i \neq j)$, represent the net interconversion rates of species $j$ in the kinetic equation of species $i$. For a system of $N$ species obeying mass conservation, the sum of the elements of each column in $\boldsymbol{K}$ must be equal to zero. In this manner, the diagonal elements can be simply defined as the negative of the sum of all other elements in the column (eq. 9):

$$
\{\boldsymbol{K}\}_{i i}=-\sum_{i=1, i \neq j}^{N}\{\boldsymbol{K}\}_{i j}
$$

The definition in eq. 9 follows from the intuitive concept that every chemical species is changing only because of interconversion to/from other species. ${ }^{34}$ An analytical solution to eq. 7 is given by: ${ }^{31}$

$$
\boldsymbol{C}(t)=\operatorname{expm}(-\boldsymbol{K} t) \cdot \boldsymbol{C}(0),
$$

where $\boldsymbol{C}(0)$ is a vector containing the initial (known) concentrations of each species, and the term expm $(-\boldsymbol{K} t)$ corresponds to a matrix exponential, explained briefly in the following section. Note the similarity between eq. 10 and the more familiar scalar case.

\section{Matrix Exponentials}

Exponentiation of scalars $[\exp (x)]$ is a familiar operation for many students, as it is introduced in elementary mathematics courses. The exponential of a matrix, in contrast, is very rarely discussed in introductory linear algebra courses.

For matrices, it is important to distinguish a matrix exponential $[\boldsymbol{P}=\operatorname{expm}(\boldsymbol{K})]$ from an element-wise exponentiation $\left[\{\boldsymbol{Q}\}_{i j}=\exp \left(\{\boldsymbol{A}\}_{i j}\right)\right]$. The former is a matrix function on square matrices, while the latter can be applied to any matrix. It follows that, in general, $\boldsymbol{P} \neq \boldsymbol{Q}$. Since in eq. 10 we involve the matrix exponential, it is worthwhile mentioning two ways to calculate it.
First, since the exponential function can be expressed as a power series, we can write it as a Taylor series considering a matrix argument:

$$
\operatorname{expm}(\boldsymbol{K})=\sum_{n=0}^{\infty} \frac{1}{n !} \boldsymbol{K}^{n}, \quad \text { with } \quad \boldsymbol{K}^{0} \equiv \mathbb{I}_{N \times N},
$$

where $\mathbb{I}_{N \times N}$ is the identity matrix. For numerical calculations, the series in eq. 11 can be truncated after a given number of terms depending on the desired accuracy. ${ }^{35}$

In a second method, if $\boldsymbol{K}$ is diagonalisable, there exists a matrix $\boldsymbol{U}$ and a diagonal matrix $\boldsymbol{\Lambda}$ such that:

$$
\boldsymbol{K}=\boldsymbol{U} \boldsymbol{\Lambda} \boldsymbol{U}^{-1}
$$

which leads to: ${ }^{36}$

$$
\operatorname{expm}(\boldsymbol{K})=\boldsymbol{U} \operatorname{expm}(\boldsymbol{\Lambda}) \boldsymbol{U}^{-1}
$$

For a diagonal matrix, the matrix exponential is equal to the (conventional) exponential of the elements of the diagonal. ${ }^{36}$ The interested reader can consult refs. $36-38$ for further details.

\section{From Kinetics to Spectral Changes}

Until now, we have focused in the kinetic (concentration) profiles of the different species involved in a reaction. We often monitor the spectral changes associated with the kinetic evolution of the system under study, leading us to the present description of time-resolved spectra. Under the assumption that the spectral properties of the involved species are time-independent, and that the kinetic profiles are independent of the detection channel - an assumption called bilinearity between the concentration and spectra - we can write an array of time-dependent spectra, $\boldsymbol{D}(t, \lambda)$, as the product of a species concentration matrix, $\boldsymbol{C}(t)$, and a spectral matrix, $\boldsymbol{S}(\lambda)$, as follows: ${ }^{7}$

$$
\boldsymbol{D}(t, \lambda)=\boldsymbol{C}(t) \cdot \boldsymbol{S}(\lambda)
$$

From the definitions given above, $\boldsymbol{C}(t)$ is a $k \times N$ matrix where every row corresponds to one sampled time point and every column to a different species. Similarly, $\boldsymbol{S}(\lambda)$ is an $N \times m$ matrix where every row corresponds to a species and every column represents a spectral channel (i.e. wavelength or frequency). This leads to $\boldsymbol{D}(t, \lambda)$, which is a matrix of time-resolved spectra containing $k$ time points (rows) and $m$ spectroscopic channels (columns).

As nicely explained by Vauthey and co-workers, ${ }^{7}$ the assumed bilinearity of the time-dependent spectra can break down if either the assumption of classical kinetics (i.e. well-defined species and intermediates) or the assumption of time-invariant spectra of these species do not hold.

For example, in ultrafast transient experiments, solvent relaxation and fast population kinetics can compete 

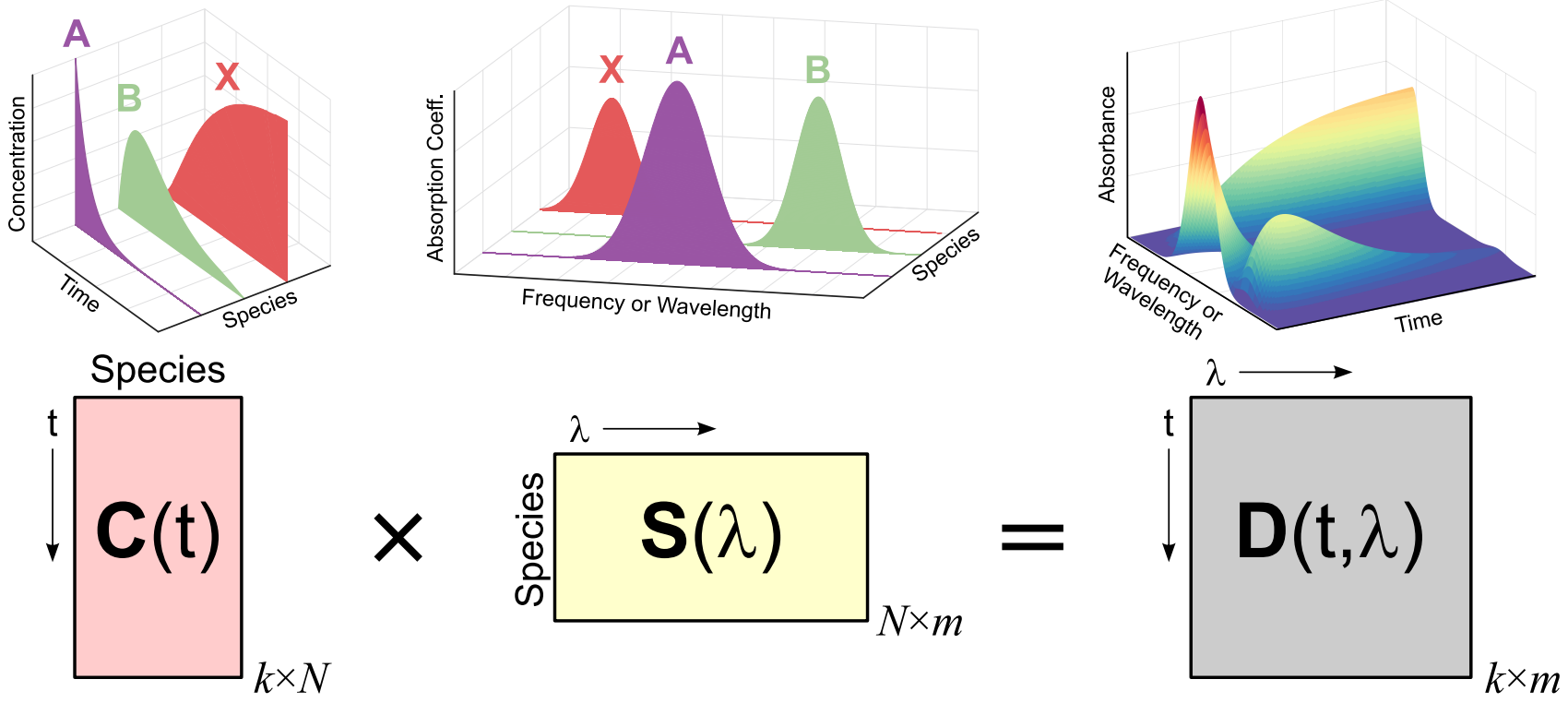

Figure 2. Visual representation of eq. 14: Under the assumption of bilinearity, the product of a matrix containing the concentration profiles $[\boldsymbol{C}(t)]$ and a matrix with the spectra of each species $[\boldsymbol{S}(\lambda)]$, yields the time-resolved absorption spectra, $\boldsymbol{D}(t, \lambda)$.

with each other. In this scenario, no clear intermediates can be defined and thus no time-invariant speciesassociated spectral features can be described. Some examples of these scenarios are nicely discussed in ref. 7, and are especially evident when studying solvation in ionic liquids. 39,40

\section{E. The Inverse Problem: From Spectral Changes to Kinetic Profiles and Mechanisms}

If we know the concentration profiles and speciesassociated spectra of a system, then calculation of the time-resolved spectra that would be observed is a trivial matter, and can be done merely by using eq. 14 .

In practice, however, we wish to solve the inverse problem: we want to obtain the concentration profiles and species-associated spectra for the system under study, given a reasonable kinetic or spectral model.

To begin our discussion on the inverse problem, we start by characterising a real experimental dataset with noise $\left(\boldsymbol{D}_{e}\right)$ in a manner analogous to eq. 14 . We calculate a parameter-dependent concentration matrix using a given kinetic model, $\boldsymbol{C}(t ; \beta)$ (where $\beta$ are the kinetic parameters), and obtain the best possible guess for a spectral matrix, $\hat{\boldsymbol{S}}(\lambda)$-where the circumflex indicates this is not an exact solution but an estimate one. ${ }^{7}$ In this manner, we can define $\boldsymbol{D}_{\text {fit }}$ as:

$$
\boldsymbol{D}_{\text {fit }}(t, \lambda ; \beta)=\boldsymbol{C}(t ; \beta) \cdot \hat{\boldsymbol{S}}(\lambda)
$$

To calculate $\hat{\boldsymbol{S}}(\lambda)$, we can take advantage of a mathematical tool called the Moore-Penrose pseudoinverse, explained in more detail in the next section.

\section{F. The Moore-Penrose Pseudoinverse: Least-Squares Fit}

The problem in eq. 15 could be analytically solved if, for example, $\boldsymbol{C}(t ; \beta)$ was a square invertible matrix.

As is shown in elementary linear algebra courses, the matrix equation $\boldsymbol{A} \boldsymbol{X}=\boldsymbol{B}$ can be solved for $\boldsymbol{X}$ if $\boldsymbol{A}$ is invertible, the solution being $\boldsymbol{X}=\boldsymbol{A}^{-1} \mathbf{B}$.

If $\boldsymbol{A}$ is square but not invertible, or if it is a rectangular matrix - as is normally the case for $\boldsymbol{C}(t)$ - then we can define a unique matrix $\boldsymbol{A}^{+}$which satisfies the MoorePenrose conditions: ${ }^{41}$

$$
\begin{aligned}
\boldsymbol{A} \boldsymbol{A}^{+} \boldsymbol{A} & =\boldsymbol{A} \\
\boldsymbol{A}^{+} \boldsymbol{A} \boldsymbol{A}^{+} & =\boldsymbol{A}^{+} \\
\left(\boldsymbol{A} \boldsymbol{A}^{+}\right)^{*} & =\boldsymbol{A} \boldsymbol{A}^{+} \\
\left(\boldsymbol{A}^{+} \boldsymbol{A}\right)^{*} & =\boldsymbol{A}^{+} \boldsymbol{A}
\end{aligned}
$$

where $(\cdots)^{*}$ denotes the conjugate transpose of a matrix. If $\boldsymbol{A}$ is invertible, then $\boldsymbol{A}^{+}=\boldsymbol{A}^{-1}$. If it is noninvertible but has linearly independent rows (eq. 17a) or columns (eq. 17b), then $\boldsymbol{A}^{+}$can be computed as: ${ }^{41}$

$$
\begin{array}{lll}
\boldsymbol{A}^{+}=\left(\boldsymbol{A}^{*} \boldsymbol{A}\right)^{-1} \boldsymbol{A}^{*}, & \text { thus } & \boldsymbol{A}^{+} \boldsymbol{A}=\mathbb{I} \\
\boldsymbol{A}^{+}=\boldsymbol{A}^{*}\left(\boldsymbol{A} \boldsymbol{A}^{*}\right)^{-1}, & \text { thus } & \boldsymbol{A} \boldsymbol{A}^{+}=\mathbb{I}
\end{array}
$$

We can use the Moore-Penrose pseudoinverse of $\boldsymbol{C}(t ; \beta)$ to calculate $\hat{\boldsymbol{S}}(\lambda)$, thus solving the problem from eq. 15 . It can be shown that $\boldsymbol{A}^{+}$leads to the least-squares fit of a linear system whenever an exact solution does not exist (i.e. $\boldsymbol{A}$ is singular or rectangular). ${ }^{41}$

Combining eq. $17 \mathrm{a}$ and eq. 14 , we have: ${ }^{42}$

$$
\boldsymbol{C}^{+} \boldsymbol{D}_{e}=\boldsymbol{C}^{+} \boldsymbol{C} \cdot \hat{\boldsymbol{S}} \text { and hence } \hat{\boldsymbol{S}}=\boldsymbol{C}^{+} \boldsymbol{D}_{e},
$$


where the variables have been omitted for clarity, and $\boldsymbol{D}_{e}$ represents the experimental dataset.

Combining eq. 15 , eq. $17 \mathrm{a}$, and eq. 18 , the calculated dataset for the chosen set of parameters $(\beta)$ is: ${ }^{43}$

$$
\boldsymbol{D}_{\text {fit }}(t, \lambda ; \beta)=\boldsymbol{C}(t ; \beta) \cdot\left[\boldsymbol{C}^{+}(t ; \beta) \cdot \boldsymbol{D}_{e}(t, \lambda)\right]
$$

Note that in this equation $\boldsymbol{C C}^{+} \neq \mathbb{I}$, since we have used the definition of $\boldsymbol{C}^{+}$from eq. 17a.

Variation of $\beta$ would allow-using a least-squares minimisation routine - to obtain the best-fitting reconstruction of the experimental time-resolved spectra, $\boldsymbol{D}_{\text {opt }}\left(t, \lambda ; \beta_{\text {opt }}\right)$, which minimises the square of norm of the residuals, $\left\|\boldsymbol{D}_{\text {fit }}(t, \lambda ; \beta)-\boldsymbol{D}_{e}(t, \lambda)\right\|^{2}$.

Note that this procedure is general and independent of the functional form of $\boldsymbol{C}(t)$, which allows for the implementation of kinetic schemes of arbitrary complexity towards the analysis of time-resolved spectra. Also important is to note that the dependence on $\beta$ is non-linear, and hence $\beta_{\text {opt }}$ must be found iteratively. ${ }^{44}$

We have included in the Supporting Information a set of fully functional MATLAB code, with a detailed description of both the code and the above procedure. We consider a generalised network of coupled first-order reactions as well as an arbitrary kinetic model defined by a system of ODEs. Simulation and fitting routines are also provided and discussed in detail.

\section{EXPERIMENTAL}

\section{A. Chemicals and Solvents}

All chemicals and reagents were used as received from their suppliers. The solutions were prepared in doublydistilled water. Stock solutions of $\mathrm{KMnO}_{4}$ in water were freshly prepared to avoid degradation upon storage.

\section{B. UV-Visible Spectrokinetic Measurements}

To acquire UV-Vis absorption spectra of the reaction mixtures, a $1 \times 1 \mathrm{~cm}$ pathlength quartz cuvette, equipped with a small magnetic stir bar was coupled to a fibre optics spectrometer (Ocean Optics USB4000, with a DH2000-BAL fibre-coupled tungsten/deuterium lamp). Optical filters before the sample cuvette were used to adapt the light intensity to the dynamic range of the detector.

Absorption spectra were recorded before and after addition of $10 \mu \mathrm{L}$ of a $700 \mathrm{~mm}(7 \mu \mathrm{mol})$ aqueous stock solution of the corresponding sugar ( $D$-glucose, $D$-fructose or sucrose) to a freshly prepared reaction mixture containing $1.4 \mathrm{~mL}$ dd $-\mathrm{H}_{2} \mathrm{O}, 500 \mu \mathrm{L}$ of $400 \mathrm{~mm} \mathrm{NaOH}(200 \mu \mathrm{mol})$, and $1 \mathrm{~mL}$ of $0.5 \mathrm{mM} \mathrm{KMnO}_{4}(0.5 \mu \mathrm{mol})$. The cuvette was stirred at all times to minimise the mixing time. The initial concentrations of the sugar, $\mathrm{NaOH}$ and $\mathrm{KMnO}_{4}$ were equal to $2.4,68.7$ and $0.2 \mathrm{~mm}$, respectively. Doublydistilled water was used to record the intensity background (blank spectrum). All measurements were run using the OceanView 2.0 software (Ocean Insight).
The reaction was monitored until formation of the yellow/brown $\mathrm{MnO}_{2}$ species was complete and no further colour changes were evident to the naked eye. All reactions were complete within 10-30 min, depending strongly on the identity of the sugar and the starting concentrations of the reagents. The initial concentrations chosen above lead to a pseudo-first order behaviour of the bimolecular steps involving the sugar and the $\mathrm{Mn}$ species. This allows us to treat the data - to a very good approximation - with the first order kinetic models discussed herein, for a simplified pedagogical approach.

A video file which illustrates in real time the colour and spectral changes observed during a typical experimental run is included in the SI.

\section{Data Analysis}

All calculations were performed in MATLAB R2021b using home-made scripts, which build upon our previous examples of modelling arbitrary chemical kinetics. ${ }^{15}$ We have adopted a data analysis strategy based in the concepts discussed in the Theoretical Background section (section II) of this paper. Further details on the implementation of the code are given in the Supporting Information, with a detailed explanation and potential changes to the code to adapt it to a general kinetic model.

\section{RESULTS AND DISCUSSION}

\section{A. Interpreting the Spectral Changes During the Chameleon Reaction}

Contour maps of the time-dependent UV-Vis absorption spectra recorded after adding the corresponding sugar to the basic $\mathrm{KMnO}_{4}$ solution are shown in Figure 3. As is evident from the timescale of these plots, the overall reaction rates decrease in the order: fructose $\gg$ glucose $>$ sucrose. In the following we will examine the data in more detail.

We begin our dissection of the time-resolved spectra by discussing the main features evident in the contour maps (Figure 3). As indicated by Roman numerals I-III in the corresponding subplots of this figure, at least three main spectrokinetic features can be observed in all three sugars - a fourth feature clearly appearing in the fructose dataset, the sugar with the fastest overall reaction rate.

While these spectrokinetic features suggest the presence of at least three or four intermediates, a more quantitative criterion is needed before considering a mechanism. The number of (spectrally) observable species will ultimately determine the number of components $(N)$ needed to build our concentration and species matrices. An over- or underestimation in the number of components can result in spurious or physically meaningless analysis, despite a potentially good fit.

The singular value decomposition (SVD) of the array of time-resolved spectra provides a preliminary estimation on the number of spectrally observable components. 

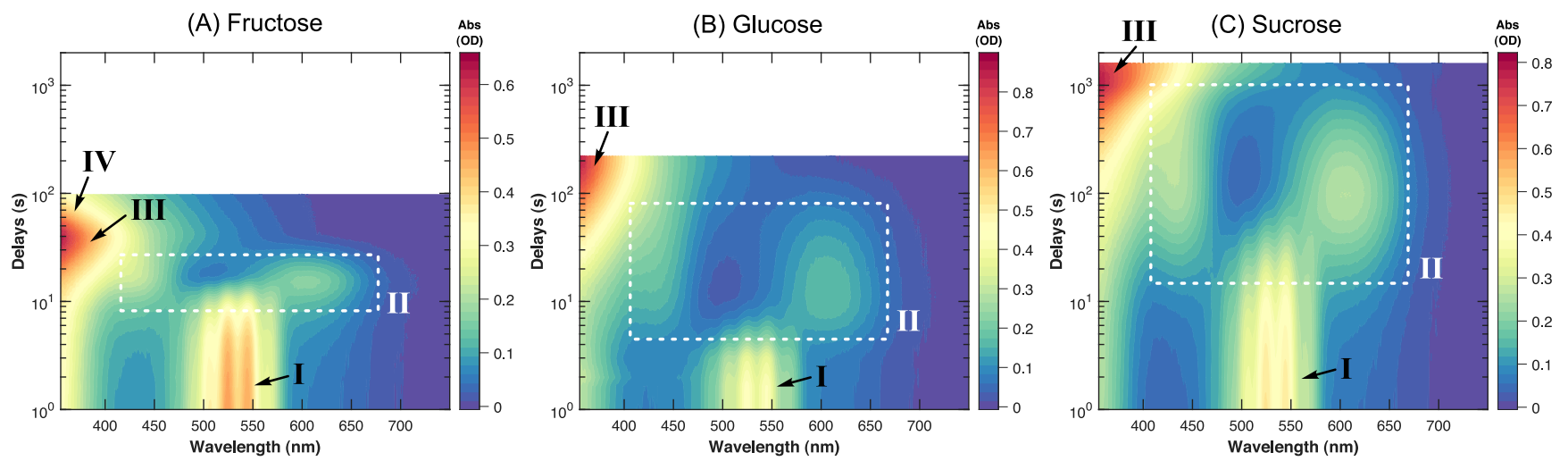

Figure 3. Spectral changes observed during kinetic runs of the oxidation of fructose $(A)$, glucose $(B)$ and sucrose $(C)$ with basic $\mathrm{KMnO}_{4}$. All datasets are plotted from 1 to $2000 \mathrm{~s}$ in a logarithmic time axis for easier comparison. Roman numerals identify the main spectrokinetic features evident from the contour plots, see text for discussion.

This mathematical decomposition expresses a real matrix $(\boldsymbol{D})$ as the product of a diagonal matrix $(\boldsymbol{\Sigma})$ and two real orthogonal matrices, $\boldsymbol{U}$ and $\boldsymbol{V}$ as follows:

$$
\boldsymbol{D}=\boldsymbol{U} \boldsymbol{\Sigma} \boldsymbol{V}^{\mathrm{T}}
$$

where the columns of $\boldsymbol{U}$ and $\boldsymbol{V}$ are known, respectively, as the left- and right-singular vectors, and are related to the time $(\boldsymbol{U})$ and spectral $(\boldsymbol{V})$ components. The diagonal elements of $\boldsymbol{\Sigma}$ are known as the singular values of $\boldsymbol{D}$ (denoted as $\sigma_{i}$ ). Choosing the decomposition as to sort the singular values in descending order, this factorization is unique up to a unitary transformation. SVD constitutes a generalisation of the eigenvalue decomposition (diagonalisation), the former being also applicable to rectangular matrices. In addition, the Moore-Penrose pseudoinverse is related to the SVD by: ${ }^{37}$

$$
\boldsymbol{D}^{+}=\boldsymbol{V} \boldsymbol{\Sigma}^{+} \boldsymbol{U}^{\mathrm{T}},
$$

where $\left\{\boldsymbol{\Sigma}^{+}\right\}_{i j}=1 /\{\boldsymbol{\Sigma}\}_{i j}$ for every non-zero diagonal element. Whenever eq. 14 holds, the magnitude of the singular values gives an estimate on the number of components. For a noise-free dataset, the number of nonzero $\sigma_{i}$ is equal to $N$. In the presence of noise, there exists a cut-off point between significant $\sigma_{i}$ and the noise level. This is illustrated in Figure 4 for our three datasets. We observe that the first three/four $\sigma_{i}$ 's are significantly higher than the rest (the vertical axis in Figure 4 is logarithmic), and that the magnitude of the following $\sigma_{i}$ 's decays roughly in an exponential manner with the index $i$ (evidenced by a straight line in Figure 4). ${ }^{1}$

Having estimated the number of components, we then move on to a discussion of plausible reaction mechanisms, to ultimately fit our data to a kinetic model and to elucidate the spectra of the individual species.

\section{B. Proposing a Reaction Mechanism}

Permanganate is an important oxidant in many organic and inorganic redox transformations. ${ }^{45}$ Amongst

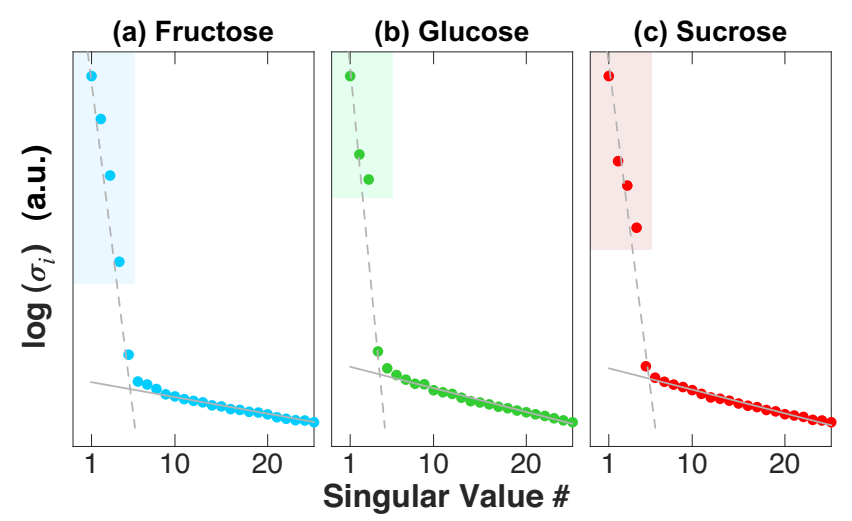

Figure 4. Relative magnitudes of the first 25 singular values of the fructose (A), glucose (B) and sucrose (C) datasets. Lines to guide the eye show the noise level (solid lines) and significant/non-noise singular values (dashed lines), the latter highlighted by coloured boxes.

the organic substrates that can be oxidised by permanganate, we will focus our attention on carbohydrates (sugars). Several mechanisms have been proposed for both acidic, ${ }^{46}$ and basic aqueous media, ${ }^{47,48}$ which are complex and depend on the concentration of both reactants, and the $\mathrm{pH}$ of the medium. ${ }^{45,47,49-53}$ An in-depth examination of such mechanisms is outside the scope of this work. The interested reader can find more details in ref. 45 .

The first mechanism we will discuss is shown in eq. $22:^{54}$

$$
\begin{aligned}
& \mathrm{MnO}_{4}^{-}+\mathrm{e}^{-} \stackrel{k_{1}^{\prime}}{\longrightarrow} \mathrm{MnO}_{4}^{2-} \\
& \mathrm{MnO}_{4}^{2-}+\mathrm{e}^{-} \stackrel{k_{2}^{\prime}}{\longrightarrow} \mathrm{MnO}_{4}^{3-} \\
& 2 \mathrm{MnO}_{4}^{3-} \stackrel{k_{3}^{\prime}}{\longrightarrow} \mathrm{MnO}_{4}^{2-}+\mathrm{Mn}_{(\mathrm{aq})}^{(\mathrm{IV})} \\
& \mathrm{Mn}_{(\mathrm{aq})}^{(\mathrm{IV})} \stackrel{k_{4}^{\prime}}{\longrightarrow} \mathrm{MnO}_{2} \downarrow
\end{aligned}
$$

The first step (eq. 22a) represents the initial reduc- 
tion of permanganate $[\mathrm{Mn}(\mathrm{VII})]$ to manganate $\left[\mathrm{MnO}_{4}^{2-}\right.$, $\mathrm{Mn}(\mathrm{VI})$ ], followed by the generation of the hypomanganate $\left(\mathrm{MnO}_{4}^{3-}\right)$ anion [eq. $\left.22 \mathrm{~b}, \mathrm{Mn}(\mathrm{V})\right]$, which disproportionates to give $\mathrm{MnO}_{4}^{2-}[\mathrm{Mn}(\mathrm{VI})]$ and $\mathrm{Mn}_{(\mathrm{aq})}^{(\mathrm{IV})}$ as products (eq. 22c). The last step (eq. 22d) represents the precipitation of a soluble $\mathrm{Mn}(\mathrm{IV})$ species to form a colloidal suspension of $\mathrm{MnO}_{2}$, as reported in literature. ${ }^{55}$ This mechanism would lead to the following ODEs:

$$
\left\{\begin{aligned}
\frac{d\left[\mathrm{MnO}_{4}^{-}\right]_{t}}{d t} & =-k_{1}^{\prime}\left[\mathrm{MnO}_{4}^{-}\right]_{t} \\
\frac{d\left[\mathrm{MnO}_{4}^{2-}\right]_{t}}{d t} & =+k_{1}^{\prime}\left[\mathrm{MnO}_{4}^{-}\right]_{t}-k_{2}^{\prime}\left[\mathrm{MnO}_{4}^{2-}\right]_{t} \\
\frac{d\left[\mathrm{MnO}_{4}^{3-}\right]_{t}}{d t} & =+k_{2}^{\prime}\left[\mathrm{MnO}_{4}^{2-}\right]_{t}-k_{3}^{\prime}\left[\mathrm{MnO}_{4}^{3-}\right]_{t}^{2} \\
\frac{d\left[\mathrm{Mn}_{(\mathrm{aq})}^{(\mathrm{IV})}\right]_{t}}{d t} & =+k_{3}^{\prime}\left[\mathrm{MnO}_{4}^{3-}\right]_{t}^{2}-k_{4}^{\prime}\left[\mathrm{Mn}_{(\mathrm{aq})}^{(\mathrm{IV})}\right]_{t} \\
\frac{d\left[\mathrm{MnO}_{2}\right]_{t}}{d t} & =+k_{4}^{\prime}\left[\mathrm{Mn}_{(\mathrm{aq})}^{(\mathrm{IV})}\right]_{t}
\end{aligned}\right.
$$

The $\mathrm{MnO}_{4}^{3-}$ species is highly unstable in basic media. ${ }^{47,56-58}$ Hence, we can consider an alternative mechanism where the $\mathrm{MnO}_{4}^{2-}$ species undergoes a twoelectron reduction instead, leading directly to the soluble $\mathrm{Mn}(\mathrm{IV})$ species, which then precipitates as before (eq. 24):

$$
\begin{array}{r}
\mathrm{MnO}_{4}^{-}+\mathrm{e}^{-} \stackrel{k_{1}}{\longrightarrow} \mathrm{MnO}_{4}^{2-} \\
\mathrm{MnO}_{4}^{2-}+2 \mathrm{e}^{-} \stackrel{k_{2}}{\longrightarrow} \mathrm{Mn}_{(\mathrm{aq})}^{(\mathrm{IV})} \\
\mathrm{Mn}_{(\mathrm{aq})}^{(\mathrm{IV})} \stackrel{k_{3}}{\longrightarrow} \mathrm{MnO}_{2 \downarrow}
\end{array}
$$

This mechanism leads to following ODE system:

$$
\left\{\begin{aligned}
\frac{d\left[\mathrm{MnO}_{4}^{-}\right]_{t}}{d t} & =-k_{1}\left[\mathrm{MnO}_{4}^{-}\right]_{t} \\
\frac{d\left[\mathrm{MnO}_{4}^{2-}\right]_{t}}{d t} & =+k_{1}\left[\mathrm{MnO}_{4}^{-}\right]_{t}-k_{2}\left[\mathrm{MnO}_{4}^{2-}\right]_{t} \\
\frac{d\left[\mathrm{Mn}_{(\mathrm{aq})}^{(\mathrm{IV})}\right]_{t}}{d t} & =+k_{2}\left[\mathrm{MnO}_{4}^{2-}\right]_{t}-k_{3}\left[\mathrm{Mn}_{(\mathrm{aq})}^{(\mathrm{IV})}\right]_{t} \\
\frac{d\left[\mathrm{MnO}_{2}\right]_{t}}{d t} & =+k_{3}\left[\mathrm{Mn}_{(\mathrm{aq})}^{(\mathrm{IV})}\right]_{t}
\end{aligned}\right.
$$

The main difference between eq. 23 and eq. 25 is the absence of second-order concentration terms (e.g. $\left[\mathrm{MnO}_{4}^{3-}\right]_{t}^{2}$ in eq. $\left.23 \mathrm{c}-\mathrm{d}\right)$. Considering the pedagogical aim of our present paper, and the aforementioned instability of the $\mathrm{MnO}_{4}^{3-}$ species, we shall focus our attention on the second mechanism (eq. 24-25). Further details about this mechanism are discussed in the SI.

Defining the concentration vector as

$\boldsymbol{C}(\boldsymbol{t})=\left(\left[\mathrm{MnO}_{4}^{-}\right]_{t},\left[\mathrm{MnO}_{4}^{2-}\right]_{t},\left[\mathrm{Mn}_{(\mathrm{aq})}^{(\mathrm{IV})}\right]_{t}, \quad\left[\mathrm{MnO}_{2}\right]_{t}\right)^{\mathrm{T}}$, the rate matrix becomes:

$$
\boldsymbol{K}_{\mathrm{M} 2}=\left(\begin{array}{cccc}
-k_{1} & 0 & 0 & 0 \\
+k_{1} & -k_{2} & 0 & 0 \\
0 & +k_{2} & -k_{3} & 0 \\
0 & 0 & +k_{3} & 0
\end{array}\right)
$$

In the following section, we discuss the results of analysing our data with this kinetic model.

\section{Species Associated Spectra and Kinetic Profiles}

The ultimate goal of the analysis of time-resolved spectra is the obtention of rate constants and speciesassociated spectra (SAS) that describe the system in a physically meaningful way. In agreement with our discussion in the context of SVD (Figure 4), we have fitted the glucose dataset with three components, since we believe that our detection window did not allow us to observe the last step of the mechanism (eq. 24c). The sucrose dataset was also fit with three components, as this gave the best results. The fructose dataset was fit to a model with four components. This shows that the number of significant $\sigma_{k}$ 's is not an absolute criterion as to the number of observable components.

In Figure 6 and Table I, we show the results of fitting all three datasets (fructose, glucose and sucrose) with the model described in eq. 24 and eq. 26. More detailed plots can be found in Figures S5-S7 in the SI. The SAS obtained for all datasets can be compared with the reported spectra of the different manganese-oxy species with oxidation states of manganese ranging from VII to IV (Figure 5), adapted from ref. 57:

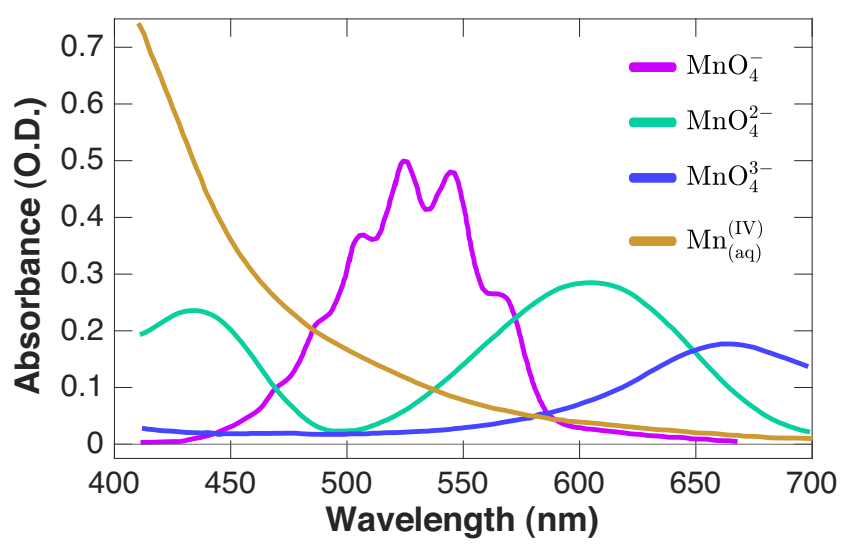

Figure 5. UV-Vis spectra of the manganese species in oxidation states VII-IV. Spectra from a $0.2 \mathrm{~mm}$ solution in a $1 \mathrm{~cm}$ cuvette at $20{ }^{\circ} \mathrm{C}$. Digitalised and adapted with permission from ref. 57. Copyright 1985 American Chemical Society.

A very important modification to the model was introduced to make the concentration of the initial species (Species 1, corresponding to $\mathrm{MnO}_{4}^{-}$) non-zero at negative reaction times. We set it to 1 as this would then lead to relative concentrations for all species. This is done to take into account that $\mathrm{MnO}_{4}^{-}$is already in the reaction mixture, and its concentration is expected to decrease only after addition of the reducing sugar-hence starting the reaction. Furthermore, to correctly fit the fructose dataset, a non-negativity penalty of the SAS was introduced (further details are given in the SI). 
(A) Fructose
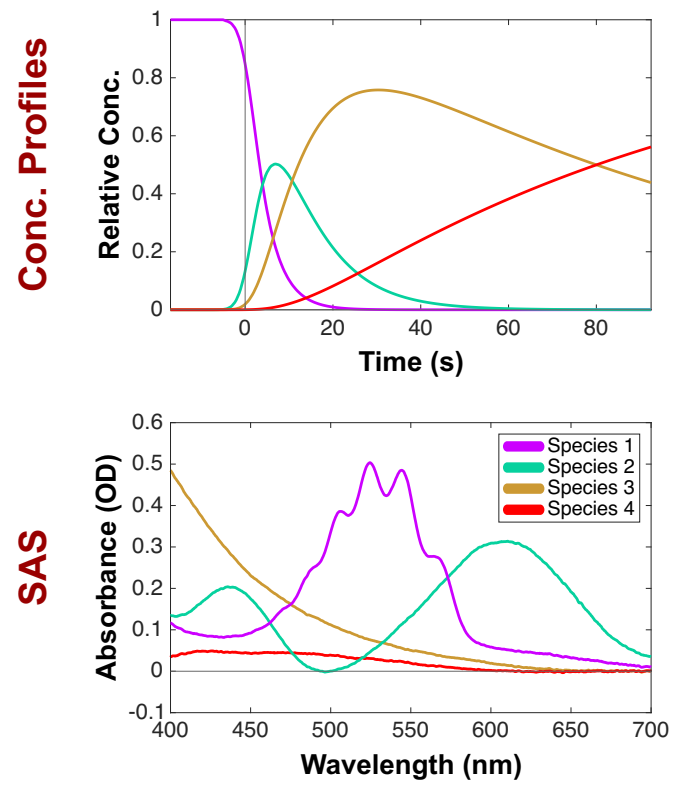

(B) Glucose
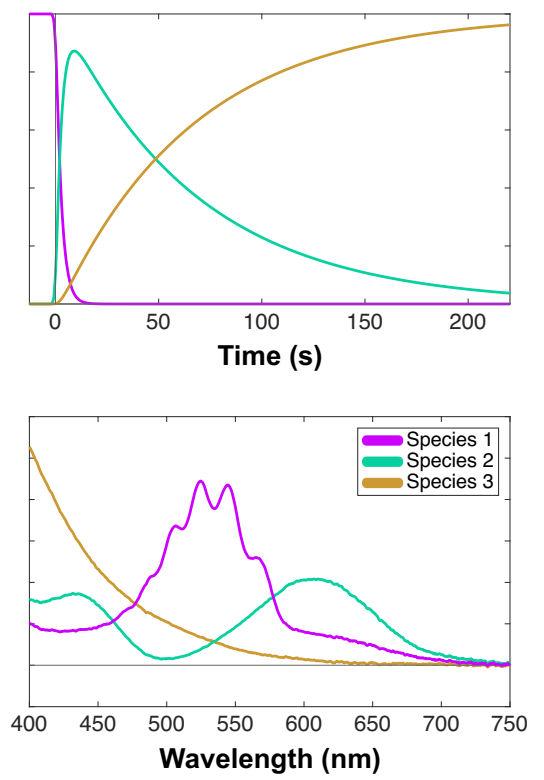

(C) Sucrose
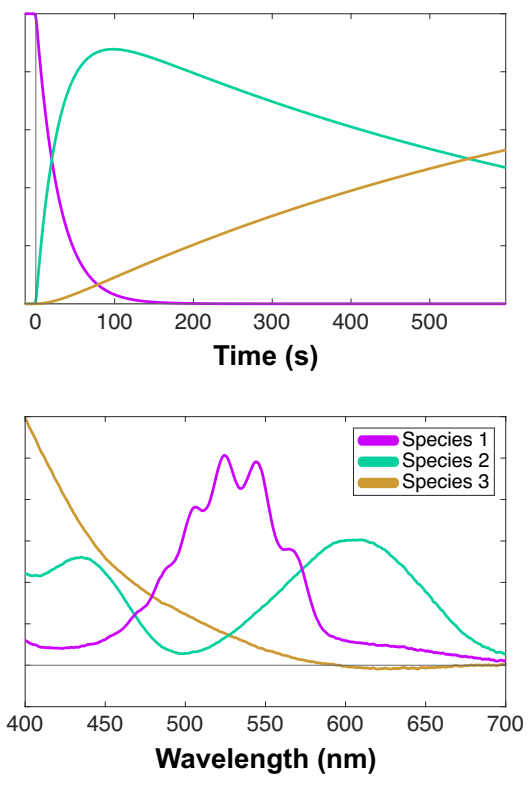

Figure 6. Concentration profiles (top row) and species-associated spectra (SAS, bottom row) obtained from the fits of the experimental datasets.

When treating time-resolved difference spectra, this needs to be considered more carefully, since speciesassociated difference spectra (SADS) can also have negative contributions (see the SI for an extended discussion). ${ }^{7}$

The fructose dataset required the inclusion of the nonnegative SAS penalty function and very careful selection of the initial parameters to converge to a physically meaningful solution, yet the quality of the fit (especially at early times) is not optimal. We believe that improper mixing and fast dynamics contribute to a non-Gaussian instrument response function (IRF), evidenced by the larger residuals appearing at early times (Figure S6 in the SI). This serves to exemplify that a reasonable fit does not necessarily imply that a given model/mechanism accurately describes the physical phenomenon under study, and careful examination of the residuals (which should be structureless) can reveal any inconsistencies.

The SAS of the fourth component (Figure 6A; which corresponds to precipitated $\mathrm{MnO}_{2}$ nanoparticles) shows a very broad, weak and featureless band. We believe that, since the formation of $\mathrm{MnO}_{2}$ nanoparticles is an uncontrolled process under our experimental conditions, the size of these nanoparticles (and hence their absorption spectra) will change over time. ${ }^{59-61}$ This last observation would then help explain why the model does not fit the data to the same degree as with the other sugars.

The sucrose dataset, on the other hand, was truncated at $t<600 \mathrm{~s}$ for the fit, since precipitation of $\mathrm{MnO}_{2}$ and degradation of $\mathrm{MnO}_{4}^{-}$by other side reactions not considered here can play a significant role and alter the kinetics at longer times.

As shown in Table I, the extracted rate constants obey,
TABLE I. Kinetic parameters of the permanganate oxidation of sugars in basic aqueous medium at room temperature

\begin{tabular}{clccc}
\hline Parameter & $($ Units $)$ & Fructose & Glucose & Sucrose \\
\hline$t_{0}$ & $(\mathrm{~s})$ & 5.72 & 2.21 & 3.57 \\
$\Delta^{\mathrm{a}}$ & $(\mathrm{s})$ & 4.96 & 1.02 & 2.17 \\
$k_{1}$ & $\left(\mathrm{~s}^{-1}\right)^{\mathrm{b}}$ & $2.33 \times 10^{-1}$ & $3.11 \times 10^{-1}$ & $3.46 \times 10^{-2}$ \\
$k_{2}$ & $\left(\mathrm{~s}^{-1}\right)^{\mathrm{b}}$ & $9.86 \times 10^{-2}$ & $1.54 \times 10^{-2}$ & $1.33 \times 10^{-3}$ \\
$k_{3}$ & $\left(\mathrm{~s}^{-1}\right)^{\mathrm{b}}$ & $1.29 \times 10^{-2}$ & - & - \\
\hline
\end{tabular}

${ }^{a}$ Full-width at half-maximum of the Gaussian IRF used to convolve the kinetic response.

$\mathrm{b}$ The kinetics of the system are being approximated by a pseudo-first-order mechanism, and as such, the rates shown herein are observed rates and will depend on concentration. See discussion of the kinetic model in the SI.

in general, the relationship $k_{1}>k_{2}>k_{3}$, indicating that the successive electron transfer steps are slower in nature, as is expected when the manganate ions lose their oxidising power (driving force). Furthermore, $k_{2}$ decreases in the order fructose $>$ glucose $>$ sucrose, becoming roughly an order of magnitude slower every time.

These results are all in agreement with the qualitative observations about the overall speed of the reaction. The initial electron transfer rates are similar for glucose and fructose, but significantly slower for sucrose. This observation can be explained by considering that sucrose is a dimer of glucose and fructose, linked through the anomeric carbon. Hence, sucrose is the only nonreducing sugar, since it has no open chain form. This makes the latter significantly more difficult to oxidise, and thus slows down the reaction. Further mechanistic details can be found elsewhere. ${ }^{45}$ 
The SAS obtained from the fits to all three datasets using the kinetic model from eq. 23 almost quantitatively match the reported spectra of the Mn-oxy species (Figure 5), confirming their identities in the kinetic model and validating the proposed reaction mechanism.

As shown in Figure 3 and Figure 6-5, and in agreement with our previous discussion about the mechanism, the $\mathrm{MnO}_{4}^{3-}$ species was not detected in our experiments. This is evidenced both by the lack of additional kinetic features at ca. $660 \mathrm{~nm}$, and by the absence of any significant absorption bands beyond $700 \mathrm{~nm}$ in all datasets.

The shapes of the SAS and the reported spectra (Figure 5) can be discussed during elementary inorganic and general chemistry courses in the context of the analysis of electronic (UV-Vis) absorption spectra. In this manner, students and lecturers can correlate the observed spectra and the position of the bands with the oxidation states and electronic configurations of the metal centre in each species (i.e. in the context of ligand field theory, see chapter 8 of ref. 62 ).

\section{Pedagogical Approach to this Paper: A Flow Chart to Analyse Time-Resolved Spectroscopic Data}

To summarise the data analysis strategies presented in this paper, we introduce the flow chart shown in Scheme 1. This scheme illustrates step-by-step the processes and subprocesses involved in each stage of data analysis of a time-resolved spectral dataset following a model-based strategy. We believe that the use of a flow chart can ease the explanation of every step in a lecture, and also serve as reference for students and lecturers, to be used in both teaching and research laboratories.

The flow chart can be explained as follows: The data is first loaded and examined into a suitable data processing software (e.g. MATLAB). The kinetic and spectral traces, as well as the contour (2D) and SVD plots are done in order to determine the $N$ components and the approximate timescales of the spectral changes. After a literature research, a kinetic model/mechanism can be proposed and fitted to the data until convergence is achieved (typically meaning that the norm of the residuals is below a certain threshold value).

A careful analysis of the extracted SAS/EAS ${ }^{63}$ and rate constants will then lead to the question of whether these results are physically meaningful - for instance, by checking the magnitude of the rate constants, the shape of the SAS/EAS, and their relationship with the expected intermediates according to the proposed kinetic model and/or literature information). If the results are not satisfactory, new starting conditions or a new mechanism/kinetic model must be tested, repeating the process over again.

The information presented in this manuscript can be used by lecturers to explain the theory behind generalised matrix kinetics for first-order reaction systems (complementary to the approach discussed by Berberan-

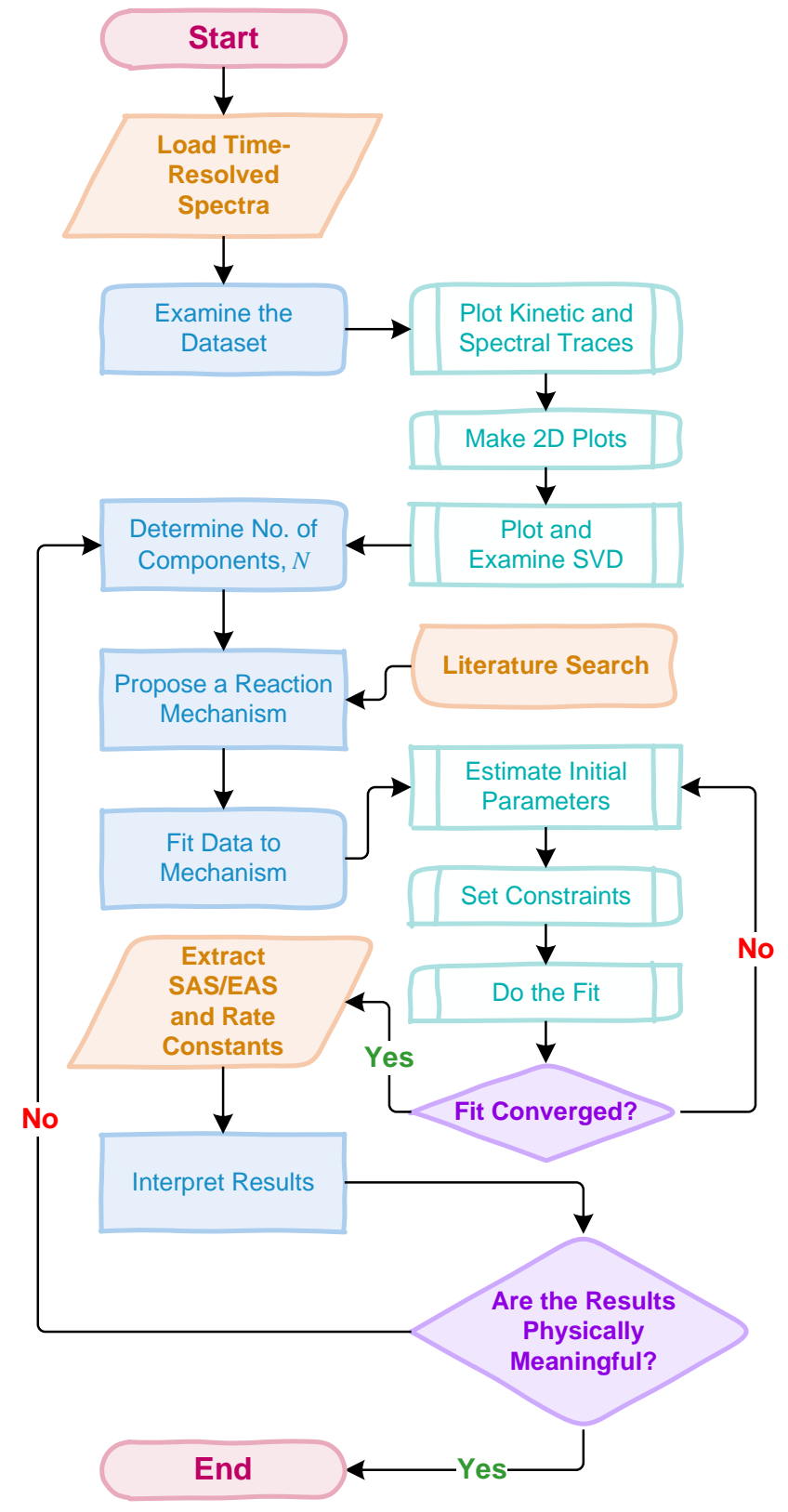

Scheme 1. Flow chart summarising a model-based data analysis strategy for time-resolved spectra.

Santos and Martinho), ${ }^{31}$ the correlation between kinetics and spectral changes, and how to propose and test reaction mechanisms (e.g. estimation of the number of components from SVD, simulation of time-resolved data, amongst others).

Our approach is versatile, and the topics discussed in this paper can be presented in several ways. For example, the lecturer can use this article in a traditional lecture as a part of a kinetics course, and then ask the students to analyse the provided datasets using our flow chart, paving the way for discussion about the possible mechanisms. As an alternative, the chemical chameleon reaction can also be implemented as a hands-on experiment, 
allowing the students to analyse and process their own data, collected by using inexpensive chemicals and equipment that is readily available in most chemistry laboratories. With a larger class, the effects of other variables such as $\mathrm{pH}$, temperature and concentration can be used to test and discuss the results and their impact in the kinetics and involved chemical mechanisms. The lecturer can then ask the students to provide enough evidence to support their own proposed mechanisms and discuss the outcome, the goodness of the fit, and the limitations of the used kinetic models.

The vast amount of information available in literature regarding time-resolved analysis can be overwhelming for an upper-division undergraduate or for graduate students beginning their research. Our manuscript can be used for a first, solid approach to the treatment of time-resolved data and kinetic analysis. These students can perform the chemical chameleon reaction on their own and then analyse the data themselves by following the step-by-step flow chart; while getting deeper knowledge about the theory behind such analysis in a facile way. The present work can also be used by senior researchers as a first activity assigned to new students, allowing them to gain experience in the collection of time-resolved data and their analysis.

\section{CONCLUSIONS}

In conclusion, herein we present a sweet introduction to the treatment of time-resolved data, exemplified by the permanganate oxidation of sugars in aqueous basic media. The mathematical aspects of a model-based data analysis strategy under the assumptions of classical kinetics and bilinearity between concentrations and spectra are discussed in detail, presented in a pedagogical manner, and summarised in a flow chart. Example code files provide a fully functional implementation of the described methods, while giving the readers the option to simulate and model their own data in a simple manner. We believe that a basic knowledge programming and data processing strategies is essential for chemists, as they can significantly facilitate the analysis of data collected on a day-to-day basis - especially true in the physical and analytical chemistry subdisciplines.

Our mechanistic discussion of this reaction bridges important concepts amongst the organic, inorganic, physical and analytical branches of chemistry-illustrating how a complex reaction can be analysed from several perspectives to gain a deeper insight. At the same time, our work provides an easy to follow treatment of the complex aspects at the interface between spectroscopy and kinetics, essential for mechanistic understanding and modelling of complex chemical systems in all timescales.

\section{ASSOCIATED CONTENT}

\section{Supporting Information}

The Supporting Information is available free of charge at http://pubs.acs.org/doi/10.1021/xxxxx.

- Detailed explanation of all MATLAB code files, an in-depth discussion of our implementation, a discussion of the kinetic model and the analogy with transient absorption spectroscopy, as well as additional figures. (PDF)

- MATLAB source code files containing the routines to simulate and fit kinetic and spectrokinetic datasets. (ZIP)

- Fructose, Glucose and Sucrose time-resolved spectral datasets. (ZIP)

- Video of an experimental run of the chameleon reaction with glucose, illustrating the real-time colour changes easily observable by naked eye. (MP4)

\section{AUTHOR INFORMATION}

\section{Corresponding Author}

* E-mail: Ricardo.Fernandez@sheffield.ac.uk and Ricardo.FernandezTeran@gmail.com

\section{ORCID identifiers}

Ricardo J. Fernández-Terán

Estefanía Sucre-Rosales

Lorenzo Echevarria

Florencio E. Hernández

0000-0002-4665-3520

0000-0002-5486-4264

0000-0002-9872-1126

0000-0001-7753-6995

\section{Notes}

The authors declare no competing financial interest.

\section{ACKNOWLEDGMENTS}

We thank our students for being our source of inspiration. We thank Prof. Eric Vauthey and Dr. James Shipp for helpful discussions. R.F.-T. thanks Prof. Isaura De Sanctis for introducing this experiment during the undergraduate general chemistry labs, and for sparking the passion for inorganic chemistry and spectroscopy through colourful reactions.

\section{REFERENCES AND NOTES}

(1) I. H. Van Stokkum, D. S. Larsen, and R. Van Grondelle, Global and target analysis of time-resolved spectra, Biochim. Biophys. Acta - Bioenerg. 1657, 82 (2004).

(2) C. Slavov, H. Hartmann, and J. Wachtveitl, Implementation and evaluation of data analysis strategies for timeresolved optical spectroscopy, Anal. Chem. 87, 2328 (2015). 
(3) W. H. Lawton and E. A. Sylvestre, Self modeling curve resolution, Technometrics 13, 617 (1971).

(4) J. Jaumot, R. Gargallo, A. De Juan, and R. Tauler, A graphical user-friendly interface for MCR-ALS: A new tool for multivariate curve resolution in MATLAB, Chemom. Intell. Lab. Syst. 76, 101 (2005).

(5) C. Ruckebusch and L. Blanchet, Multivariate curve resolution: A review of advanced and tailored applications and challenges, Anal. Chim. Acta 765, 28 (2013).

(6) J. Jaumot, A. de Juan, and R. Tauler, MCR-ALS GUI 2.0: New features and applications, Chemom. Intell. Lab. Syst. 140, 1 (2015).

(7) J. S. Beckwith, C. A. Rumble, and E. Vauthey, Data analysis in transient electronic spectroscopy - an experimentalist's view, Int. Rev. Phys. Chem. 39, 135 (2020).

(8) P. W. Atkins, J. De Paula, and J. Keeler, Atkins' Physical Chemistry, 11th ed. (Oxford University Press, Oxford, 2018).

(9) M. Soustelle, An Introduction to Chemical Kinetics, 1st ed. (Wiley, London, 2011).

(10) P. L. Houston, Chemical Kinetics and Reaction Dynamics, 1st ed., Dover Books on Chemistry (Dover Publications, New York, 2006).

(11) R. R. Pavlis, Kinetics without steady state approximations, J. Chem. Educ. 74, 1139 (1997).

(12) Stiffness, in the context of systems of ODEs, refers to the numerical instability of certain solution methods, where a very small (often unfeasible) step size would be required to obtain a reasonable solution.

(13) D. F. Griffiths and D. J. Higham, Numerical Methods for Ordinary Differential Equations, 1st ed., Springer Undergraduate Mathematics Series (Springer-Verlag, London, 2011).

(14) J. D. Lambert, Numerical Methods for Ordinary Differential Systems: The Initial Value Problem, 1st ed. (Wiley, 1991).

(15) E. Sucre-Rosales, R. Fernández-Terán, D. Carvajal, L. Echevarría, and F. E. Hernández, Experience-Based Learning Approach to Chemical Kinetics: Learning from the COVID-19 Pandemic, J. Chem. Educ. 97, 2598 (2020).

(16) R. Fernández-Terán, E. Sucre-Rosales, L. Echevarría, and F. E. Hernández, Social Distancing During the COVID-19 Pandemic: An Analogy to Explain Collision Cross-Sections in Chemical Kinetics, J. Chem. Educ. 97, 4540 (2020).

(17) A. D. Corlan and J. Ross, Kinetics methods for clinical epidemiology problems, Proc. Natl. Acad. Sci. U. S. A. 112, 14150 (2015).

(18) F. Manenti, A. Galeazzi, F. Bisotti, K. Prifti, A. Dell'Angelo, A. Di Pretoro, and C. Ariatti, Analogies between SARS-CoV-2 infection dynamics and batch chemical reactor behavior, Chem. Eng. Sci. 227, 115918 (2020).

(19) C. M. Simon, The SIR dynamic model of infectious disease transmission and its analogy with chemical kinetics, PeerJ Phys. Chem. 2, e14 (2020).

(20) G. C. McBane, SIR (Susceptible-Infectious-Removed) Model of Epidemiology as an Extended Example for Chemical Kinetics Students, J. Chem. Educ. 98, 2906 (2021).

(21) N. Weder, B. Probst, L. Sévery, R. J. Fernández-Terán, J. Beckord, O. Blacque, S. D. Tilley, P. Hamm, J. Osterwalder, and R. Alberto, Mechanistic insights into pho- tocatalysis and over two days of stable $\mathrm{H}_{2}$ generation in electrocatalysis by a molecular cobalt catalyst immobilized on $\mathrm{TiO}_{2}$, Catal. Sci. Technol. 10, 2549 (2020).

(22) K. Oppelt, M. Mosberger, J. Ruf, R. Fernández-Terán, B. Probst, R. Alberto, and P. Hamm, Shedding Light on the Molecular Surface Assembly at the Nanoscale Level: Dynamics of a $\operatorname{Re}(\mathrm{I})$ Carbonyl Photosensitizer with a Coadsorbed Cobalt Tetrapyridyl Water Reduction Catalyst on $\mathrm{ZrO}_{2}$, J. Phys. Chem. C 124, 12502 (2020).

(23) T. Liu, R. Tyburski, S. Wang, R. Fernández-Terán, S. Ott, and L. Hammarström, Elucidating ProtonCoupled Electron Transfer Mechanisms of Metal Hydrides with Free Energy- And Pressure-Dependent Kinetics, J. Am. Chem. Soc. 141, 17245 (2019).

(24) D. Buhrke, K. T. Oppelt, P. J. Heckmeier, R. FernándezTerán, and P. Hamm, Nanosecond protein dynamics in a red/green cyanobacteriochrome revealed by transient IR spectroscopy, J. Chem. Phys. 153, 245101 (2020).

(25) H. W. Roesky and K. Mockel, Chemical Curiosities (VCH, 1996).

(26) R. B. Thompson, Illustrated Guide to Home Chemistry Experiments: All Lab, No Lecture, DIY Science (O'Reilly Media, 2012).

(27) S. Senol, A. Yılmaz, and Ö. Geban, The Effects of Process Oriented Guided Inquiry Learning Environment on Students' Self-Regulated Learning Skills, Probl. Educ. 21st Century 66, 54 (2015).

(28) M. Prolongo and G. Pinto, Colourful chemistry: redox reactions with lollipops, Sci. Sch. 43, 41 (2018).

(29) A. Janonis, E. Kiudys, M. Girdžiūna, T. Blažauskas, L. Paulauskas, and A. Andrejevas, Escape the Lab: Chemical Experiments in Virtual Reality, Commun. Comput. Inf. Sci. 1283 CCIS, 273 (2020).

(30) The Chemical Chameleon - Navy Science Squad (2022).

(31) M. N. Berberan-Santos and J. M. Martinho, The integration of kinetic rate equations by matrix methods, J. Chem. Educ. 67, 375 (1990).

(32) M. N. Berberan-Santos, L. Pogliani, and J. M. Martinho, A convolution approach to the kinetics of chemical and photochemical reactions, React. Kinet. Catal. Lett. 54, 287 (1995).

(33) L. Pogliani, How to construct first-order kinetic matrices and higher-order kinetic matrices, React. Kinet. Catal. Lett. 49, 345 (1993).

(34) This assumption, however, does not always hold for a laser-based transient absorption experiment, where a population is transiently generated and hence there is a negative net flow in the population over time. That is, the excited population returns to the ground state, leading to zero concentration for all species at $t \rightarrow \infty$ (unless a permanent photoproduct is formed).

(35) In certain cases, the series will terminate after a finite number of terms. For example, if $\boldsymbol{K}$ is nilpotent, then for a certain $q>0, \boldsymbol{K}^{q}=\mathbb{O}_{N \times N}$, after which all further terms are identically zero.

(36) R. Bellman, Introduction to Matrix Analysis, 2nd ed., Classics in Applied Mathematics (Society for Industrial and Applied Mathematics, Santa Monica, California, 1997).

(37) G. H. Golub and C. F. Van Loan, Matrix Computations, 4th ed., Johns Hopkins Studies in the Mathematical Sciences (Johns Hopkins University Press, Baltimore, 2013). 
(38) B. Hall, Lie Groups, Lie Algebras, and Representations: An Elementary Introduction, 2nd ed., Graduate Texts in Mathematics (Springer International Publishing, London, 2015).

(39) X. X. Zhang, M. Liang, N. P. Ernsting, and M. Maroncelli, Conductivity and solvation dynamics in ionic liquids, J. Phys. Chem. Lett. 4, 1205 (2013).

(40) H. Marciniak and S. Lochbrunner, On the interpretation of decay associated spectra in the presence of time dependent spectral shifts, Chem. Phys. Lett. 609, 184 (2014).

(41) A. Ben-Israel and T. N. E. Greville, Generalized Inverses: Theory and Applications, 2nd ed., CMS Books in Mathematics (Springer-Verlag, New York, 2003).

(42) We use the experimental dataset, $\boldsymbol{D}_{e}$ to calculate the best-fitting $\hat{\boldsymbol{S}}$ (to minimise the norm of the residuals of $\left.\boldsymbol{D}_{e}-\boldsymbol{D}_{\text {fit }}\right)$. Using $\boldsymbol{D}_{\text {fit }}$ instead would lead to the trivial result $\boldsymbol{D}_{\text {fit }}=\boldsymbol{D}_{\text {fit }}$.

(43) Our equations are equivalent to those shown in ref. 7, except that in our case $\boldsymbol{D}$ and all other associated matrices are transposed with respect to their definition. Application of the properties of matrix multiplication yields the same results.

(44) From this discussion, it follows that eq. 19 involves alternatively finding the best set of non-linear parameters $(\beta)$ that can be transformed by a linear operator (i.e. $\boldsymbol{C} \boldsymbol{C}^{+}$) - the latter found via linear least squaresto minimise the norm of the residuals $\left(\| \boldsymbol{D}_{\text {fit }}(t, \lambda ; \beta)-\right.$ $\left.\boldsymbol{D}_{e}(t, \lambda) \|^{2}\right)$. This gives rise to the term 'alternating least squares' or ALS.

(45) J. W. Ladbury and C. F. Cullis, Kinetics and Mechanism of Oxidation by Permanganate, Chem. Rev. 58, 403 (1958).

(46) M. A. Malik, S. A. AL-Thabaiti, and Z. Khan, Kinetics of oxidation of d-glucose by permanganate in aqueous solution of cetyltrimethylammonium bromide, Colloids Surfaces A Physicochem. Eng. Asp. 337, 9 (2009).

(47) M. Jáky, Z. Szeverényi, and L. I. Simándi, Formation of manganate $(\mathrm{V})$ in oxidations by permanganate ion in strongly alkaline solutions, Inorganica Chim. Acta 186, 33 (1991).

(48) E. O. Odebunmi and S. O. Owalude, Kinetics and mechanism of oxidation of some simple reducing sugars by permanganate ion in alkaline medium, J. Iran. Chem. Soc. 5, 623 (2008).

(49) K. B. Wiberg and R. Stewart, The Mechanisms of Permanganate Oxidation. I. The Oxidation of Some Aromatic Aldehydes, J. Am. Chem. Soc. 77, 1786 (1955).

(50) R. Stewart, The Mechanisms of Permanganate Oxidation. III. The Oxidation of Benzhydrol, J. Am. Chem. Soc. 79, 3057 (1957).

(51) J. Vepřek-šiška and V. Ettel, The mechanism of the decomposition of alkaline permanganate solutions, J. Inorg. Nucl. Chem. 31, 789 (1969).

(52) F. Freeman, Postulated intermediates and activated complexes in the permanganate ion oxidation of organic compounds, Rev. React. Species Chem. React. 1, 179 (1973).

(53) S. Dash, S. Patel, and B. K. Mishra, Oxidation by permanganate: synthetic and mechanistic aspects, Tetrahedron 65, 707 (2009).

(54) We describe the mechanisms in terms of the Mn-oxy species, ignoring the sugar, since the colour changes take place mainly due to changes in the oxidation state of the metal. We acknowledge this is an approximation, but is nevertheless needed for pedagogical reasons, since the sugars do not have a spectroscopic contribution to the recorded spectra. Furthermore, we have carried out the experiments under pseudo-first-order conditions precisely to avoid dealing with the concentrations of the sugar in the proposed mechanisms.

(55) L. I. Simándi and M. Jáky, Nature of the Detectable Intermediate in the Permanganate Oxidation of transCinnamic Acid, J. Am. Chem. Soc. 98, 1995 (1976).

(56) A. Carrington and M. C. Symons, Structure and reactivity of the oxy-anions of transition metals. Part I. The manganese oxy-anions, J. Chem. Soc. , 3373 (1956).

(57) M. Jáky, L. I. Simándi, C. R. Savage, and Z. A. Schelly, Kinetics and Mechanism of the Permanganate Ion Oxidation of Sulfite in Alkaline Solutions. The Nature of Short-Lived Intermediates, J. Am. Chem. Soc. 107, 4220 (1985).

(58) D. G. Lee and T. Chen, Reduction of Manganate(VI) by Mandelic Acid and Its Significance to Development of a General Mechanism for Oxidation of Organic Compounds by High-Valent Transition Metal Oxides, J. Am. Chem. Soc. 115, 11231 (1993).

(59) S. Castillo, W. Chemnasiri, J. Donnelly, and F. Hernandez, Determination of the Relative Antioxidant Strength of Teas Combining Metal Nanoparticles, Plasmonics, and Fluorescence: A Multidisciplinary Experiment, J. Nano Educ. 8, 71 (2017).

(60) Z. M. Huang, Q. Y. Cai, D. C. Ding, J. Ge, Y. L. Hu, J. Yang, L. Zhang, and Z. H. Li, A facile label-free colorimetric method for highly sensitive glutathione detection by using manganese dioxide nanosheets, Sensors Actuators, B Chem. 242, 355 (2017).

(61) W. Haiss, N. T. Thanh, J. Aveyard, and D. G. Fernig, Determination of size and concentration of gold nanoparticles from UV-Vis spectra, Anal. Chem. 79, 4215 (2007).

(62) B. N. Figgis and M. A. Hitchman, Ligand Field Theory and its Applications, 1st ed., Special Topics in Inorganic Chemistry (Wiley-VCH, New York, 2000).

(63) When the kinetic model is approximate or when global analysis is used, the term evolution-associated spectra (EAS) is preferred. In this case, the spectra no longer correspond to distinct chemical species but rather describe the spectral changes occurring with a given time constant. The EAS can be derived from the decayassociated spectra by a simple transformation, as described in more detail in refs. 1 and 7 . 
TOC Graphic, Synopsis and Keywords

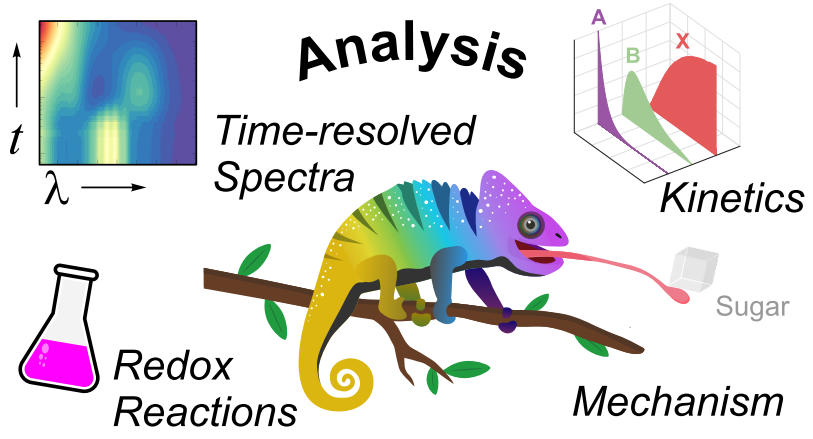

Synopsis: Herein, we present a sweet introduction to the analysis of time-resolved spectra. The so-called chameleon reaction (involving the oxidation of a sugar by $\mathrm{MnO}_{4}^{-}$in basic media) is discussed as an example system, allowing us to introduce advanced mathematical concepts required to model and fit complex kinetic mechanisms to time-resolved spectra - leading to a physically meaningful model consisting of (time-dependent) concentration profiles, and species-associated spectra.

Keywords: Upper-Division Undergraduate, Graduate Education/Research, Physical Chemistry, ComputerBased Learning, Problem Solving/Decision Making, Kinetics, Mathematics/Symbolic Mathematics, Mechanisms of Reactions, Oxidation/Reduction, Spectroscopy. 